\title{
Optimal Committee Design and Political PARTICIPATION
}

May 8, 2010

\begin{abstract}
Building on a Condorcetian common-value framework, this paper tackles the question of optimal committee formation within a finite community. Solving for the Bayesian information-aggregation game yields some interesting normative results that emphasize the presence of informational externalities as root causes of suboptimally low voluntary participation levels in communal decision-making and the Pareto-enhancing nature of a draft as compared to voluntary participation. I first derive the optimal size of a committee based on the assumption of informative voting, then I show that it is globally optimal within the class of all voting rules, and subsequently compare it to the various symmetric equilibria that may arise in a complete information setting or a Bayesian environment with heterogeneous private costs. I finally sketch out an optimal transfer scheme that may ex ante implement the socially efficient committee size.
\end{abstract}

\section{Introduction}

Following the Aristotelean designation of man as a political animal, participation in the public sphere irrespective of the structure of political authority has been regarded as a normative 
desideratum. The Ancient Greeks coined the word 'idiot' to refer in a derogatory fashion to one who manifests a flagrant lack of interest in public affairs and chooses to ensconce oneself in the private domain. Yet, the affairs of state, whether legislative, executive, or judicial, have almost always - bar few exceptions of direct democracy - been in the hands of the few. Even in the modern world, where an increasingly interdependent and uncertain environment of fragmented information and political factionalization has exacerbated the complexity of social, political, and economic organization, the norm is decision-making by committee. From national legislatures, courts, and executive cabinets down to corporate boards of directors and school boards, the quintessential tasks of creating rules, passing judgment, and solving problems are delegated to a subgroup of officials of any given community. The method of selection of who becomes a member of any committee is often dependent on the nature of its mandate, i.e., whether it primarily consists of the generation or the allocation of value across its constituents.

This paper examines the ideas of optimal participation and optimal voting institutions in political decision-making processes. How inclusive and/ or representative do these processes need to be in order to maximize social welfare? By the same token, how widespread and/ or institutionalized should the deliberation process be? The widely accepted tenet that universal participation in a decision-making process is efficient has come under dubious light of late. The most recent strand of the voting literature has brought attention to the social welfare costs of voting and deliberation thus arriving at the conclusion that minimal abstention is not desirable because of the trade-off between process inclusiveness and cost effectiveness.

In light of the above, this paper theorizes about optimal participation levels albeit within the context of a certain class of problems, namely issues of common value. The latter mostly belong to more technocratic areas of 'low politics', where the emphasis is on efficiency rather than redistribution, problem-solving rather than bargaining. I, hence, propose a model of 
information aggregation rather than preference aggregation and thus build on the results of the Condorcetian literature on juries (McLennan, 1998; Duggan and Martinelli, 2001). To that effect, I use throughout an analogous juridical terminology (comparing commonvalue decision-making problems to the trial of a defendant) without the express intention of delimiting the scope of the model to the wider class of such institutions. In other words, the terms 'jury' and 'committee' are used interchangeably denoting a finite group of people deciding over an issue over which everyone would be of the same mind given full information.

Furthermore, I adopt a mechanism design approach to complement the welfare analysis with a parsimonious attempt at institutional design. Extending this theoretical framework to a wider and more generic class of problems can help compare and contrast the implementability and efficiency of a variety of decision-making mechanisms (which span the spectrum of systems of political organization across regions and time). I seek to address such questions as when should a population faced with a decision of collective repercussions under conditions of uncertainty delegate decision-making authority to an elected subgroup of representatives (legislature), concede exclusive decision-making prerogatives to a cabinet (executive), or just vote by referendum (direct democracy)? ${ }^{1}$ Or, - counterfactually speaking -, when, if ever, would it be optimal to elect jurors and randomly draft legislators from a population? The application of tools and concepts from the field of information economics has come to bear very insightful results on similar questions of optimal design with respect to juridical (e.g., Coughlan, 2000; ?), partisan (e.g., Caillaud and Tirole, 1999, 2002), and deliberative (e.g., Meirowitz, 2006) institutions.

This set of theoretical concerns may be tackled within the context of this general family of political economy models that have come to rely upon the fascinating results of the mechanism design literature in economics. I essentially make use of the widely acclaimed results of the

\footnotetext{
${ }^{1}$ Here I do not wish to allude to issues of committee specialization or information delegation.
} 
literatures on information aggregation (see Austen-Smith and Banks, 1996; Feddersen and Pesendorfer, 1996, 1997, 1998), voter abstention (see Palfrey and Rosenthal, 1983, 1985), deliberation (Austen-Smith and Feddersen, 2005; Hafer and Landa, 2007; Meirowitz, 2007; ?), and mechanism design (e.g., Ledyard and Palfrey, 2002; ?; ?), in order to build a model of optimal committee design (namely with respect to its size and voting rule). My approach is related to that of Bulkley, Myles and Pearson (2001), Ghosal and Lockwood (2003), and Persico (2004).

In a closely related model, Persico (2004) analyzes a game of optimal committee design with costly (hence endogenous) information acquisition. Theorizing from the angle of a mechanism designer, he examines the incentives of individual jurors to acquire a noisy signal about the state of the world, wherefrom he derives the optimal number of jurors to be drafted as well as the optimal aggregation rules to be utilized. Based on the standard assumption of monotone pure strategies, Persico's main result is a necessary condition that links the optimal voting rule to the quality of available information by placing a lower bound on the range of signal accuracy that can justify the optimal voting rule. As will be done in this paper, he also makes use of the optimal aggregation rule (or statistical rule as the author calls it) in Austen-Smith and Banks (1996). Recent papers by ? and ? characterize the full set of optimal mechanisms for eliciting and aggregating information and show that in an environment of committee decision-making with costly information acquisition ex ante optimal voting schemes are not necessarily ex post efficient (i.e., from a statistical point of view) information aggregation mechanisms.

By contrast, the emphasis of this paper is on the public goods nature of communal decisionmaking in a common values environment and its resulting inefficiencies. I first derive the solution to the social planner's dual optimization problem with respect to jury size and voting rule and then contrast it to equilibria of a similar game with voluntary participation. My 
results emphasize the presence of informational externalities as root causes of suboptimally low voluntary participation levels in communal decision-making and the Pareto-enhancing nature of a draft as compared to voluntary participation. ${ }^{2}$ A further difference between my model and some of the above papers on optimal mechanism design with costly information acquisition is that this is one of exogenous information, whereby agents decide whether to participate after observing a costless signal of the state of the world. Last but not least, Persico (2004) among others disregards the social costs of voting and deliberating (which are proportional to the size of the committee) because he assumes an infinite pool of potential committee members, whilst this paper examines a similar question from the point of view of a finite community of size $N$. As a result of its finite nature, the model yields interesting normative implications linking community size to optimal decision making mechanisms. That being said, it is certainly the case that the results of the two models (Persico's and mine) converge asymptotically.

I initially sketch out the decision-making environment and basic assumptions of the model and derive the necessary optimization conditions for optimal jury design in subsection 2.1. Given that there are two policy instruments at hand, namely jury size and voting rule, I show how to make this dual optimization problem more analytically tractable. Moreover, in subsection $2.2 \mathrm{I}$ examine the comparative static and asymptotic properties of the model. Subsection 2.3 solves for the equilibria of the voluntary participation game with known costs and section 3 extends the core framework into a Bayesian environment with independent and identically distributed private costs and therefrom derives the symmetric Bayesian Nash equilibrium of the game. Subsection 3.1 delves into the mechanism design aspect of the model by gauging the social efficiency of the Bayesian equilibrium and showing how a mechanism designer (or else an external benefactor) may induce a stylized community of size $N$ to vol-

\footnotetext{
${ }^{2}$ I manage to show that by comparing optimal committee size with equilibrium participation levels.
} 
untarily achieve the efficient solution to its institutional design problem. The final section concludes by discussing some extensions to the model and stressing the similarity of the question at hand to the underprovision of public goods, since it turns out that the suboptimally low participation levels of the Bayesian game are accounted for by the presence of positive informational externalities that are ostensibly absent from the individual calculus of agents. The appendix contains the proofs not included in the text.

\section{The Basic Model}

Initially, I lay down the basic framework of the model and then comment on the expected results and implications. Assume a community with population of finite size $N>0$ (agents $i=1, \ldots, N)$ confronting a binary common value problem that uniformly affects all its members, e.g., bringing justice upon an accused defendant in a trial. First, nature assigns preferences and brings about an unknown state of the world $I$ (innocence) with prior probability $\pi \in[.5,1)$ or $G$ (guilt) with prior $1-\pi$. Then, each agent $i$ of the population receives a private independent and identically distributed signal $s_{i} \in\{i, g\}$ about the unknown state of the world with signal accuracy or informativeness $p=\operatorname{Pr}\left(s_{i}=i \mid I\right)=\operatorname{Pr}\left(s_{i}=g \mid G\right)$, where $p \in(.5,1]$. Subsequently, the community drafts an ad hoc jury of size $n$ and each jury member votes either to convict $(C)$ or acquit $(A)$ based on his/ her private information, the voting rule, and the size of the jury. Individual utilities $u$ or $u_{i}$ 's ${ }^{3}$ are defined over states and outcomes and are normalized such that $u(A, I)=u(C, G)=0, u(C, I)=-q$, and $u(A, G)=-(1-q)$, where $q \in[0,1]$. So $q$ and $1-q$ are the utility costs associated with convicting the innocent (Type I error) and acquitting the guilty (Type II error) respectively. Moreover, let us assume constant positive costs of participation and deliberation within a

\footnotetext{
${ }^{3}$ Henceforth the subscript $i$ will be dropped, since I assume no heterogeneity in preference intensities $q$ and the model is ex ante symmetric with respect to agents.
} 
jury (committee), which can be construed as individual opportunity costs $c_{i}=c>0$ (akin to voting costs in abstention models). Supposing that innocence is the status quo (hence the dictum "innocent until proven guilty"), a defendant is convicted as long as $k(n)$ or more of the jury members vote to convict (k-rule). ${ }^{4}$ Finally, let $\sigma_{i}:\{i, g\} \rightarrow[0,1]$ denote an individual voting strategy mapping private signals into the probability of voting to convict.

\subsection{Optimal Jury Size}

The mechanism designer's primary goal is to find the optimal number of jurors such that expected social welfare is maximized. Hence, one has to derive the optimal social welfaremaximizing size of the jury of size $n \in\{0,1, \ldots, N\}$ such that

$$
\begin{aligned}
n^{*} & =\underset{n \in\{0,1, \ldots, N\}}{\operatorname{argmax}} E W(\cdot, \cdot) \\
& =\underset{n \in\{0,1, \ldots, N\}}{\operatorname{argmax}}\{N[-q \pi \operatorname{Pr}(\text { Error } I ; n, k(n))-(1-q)(1-\pi) \operatorname{Pr}(\text { Error } I I ; n, k(n))]-\text { cn }\},
\end{aligned}
$$

where the objective function $E W(\cdot, \cdot)$ is the expected social welfare function, $c$ is the constant individual participation cost, $\operatorname{Pr}($ Error $I ; n, k(n))=\operatorname{Pr}(C \mid I ; n, k(n))$ and $\operatorname{Pr}($ Error $I I ; n, k(n))=$ $\operatorname{Pr}(A \mid G ; n, k(n))$ are the respective probabilities of an ex post wrong decision for given jury size $n$ and voting rule $k(n)$.

The latter probabilities obviously depend on the $k$-rule (see Feddersen and Pesendorfer, 1998) and according to the Condorcet Jury Theorem ${ }^{5}$ are also all else equal positively related to jury size (i.e., the size of the vector of i.i.d. private signals). Given the discreteness of this optimization problem and the multiplicity of plausible Nash equilibrium voting strategies

\footnotetext{
${ }^{4}$ The trivial cases of $k(n)=0, n+1$ imply a voting rule of always convicting and always acquitting respectively, regardless of committee members' signals.

${ }^{5}$ See, for example, Ladha (1992) for a discussion and formal proof of the theorem.
} 
associated with various voting rules (Feddersen and Pesendorfer, 1998), globally optimizing over both $n$ and $k(n)$ will be shown to be relatively simple on the basis of existing results in the literature. Hence, both for the sake of tractability and for reasons of economic efficiency, the scope of the model is restricted to symmetric equilibria of weakly undominated, informative voting strategies ${ }^{6}$ where each juror 'votes his signal'.

Definition 1 A voting strategy is purely informative in the above game if and only if $\sigma(i)=0$ and $\sigma(g)=1$, i.e., one votes one's signal with certainty.

Lemma 1 (Lemma 2 in Austen-Smith and Banks, 1996) For $(q, \pi, p) \in[0,1] \times[.5,1) \times(.5,1]$ and for a given integer $n$ such that $0 \leq n \leq N$, informative voting in this model is rational (i.e., a Nash equilibrium voting profile) if and only if the aggregation rule is such that $k(n)=$ $k^{*}(n)$.

Lemma 2 in Austen-Smith and Banks (1996) shows that in a similar environment informative voting is rational, that is sincere voting constitutes a Nash equilibrium profile, if and only if the $k$-rule is the optimal aggregation rule $k^{*}(n)$. Assuming that $k(n)$ denotes the number of $g$ signals among $n$ jurors, i.e., $k(n)=\#\left\{s_{i}: s_{i}=g, \forall i \in n\right\}$, then for informative voting to be rational $k^{*}(n)$ must be such that it is optimal to vote your signal given that your vote is pivotal and everyone else has voted informatively, i.e., there are $k^{*}(n)-1$ guilty signals out of $n-1$. As the above authors show formally, integer $k^{*}(n)$, where $0 \leq k^{*}(n) \leq n,{ }^{7}$ is the critical number of signals such that it is rational (i.e., a best-response strategy) to vote

\footnotetext{
${ }^{6}$ These fall under the general class of weakly undominated, responsive, and symmetric strategy profiles. Feddersen and Pesendorfer (1998, p. 26) use the term 'responsive' in reference to strategy profiles "in which jurors change their vote as a function of their private information with positive probability."

${ }^{7}$ The definition of a $k$-rule also implies that, for all $n=0,1, \ldots, N, k^{*}(n)=0$ denotes an optimal voting rule of always convicting and $k^{*}(n)=n+1$ denotes an optimal voting rule of always acquitting. See footnote below for an assumption that rules out such trivial cases.
} 
to convict based on a guilty signal and a pivotal vote, i.e.,

$$
\begin{aligned}
& E\left[u_{i}(C, \cdot) \mid \text { piv } ; s_{i}=g\right] \geq E\left[u_{i}(A, \cdot) \mid \text { piv } ; s_{i}=g\right] \\
& \quad-q \operatorname{Pr}\left(I \mid k(n)=k^{*}\right) \geq-(1-q) \operatorname{Pr}\left(G \mid k(n)=k^{*}\right)
\end{aligned}
$$

and to acquit based on an innocent signal given that your vote is pivotal, i.e.,

$$
\begin{aligned}
E\left[u_{i}(A, \cdot) \mid \text { piv } ; s_{i}=i\right] & \geq E\left[u_{i}(C, \cdot) \mid \text { piv; } s_{i}=i\right] \\
-(1-q) \operatorname{Pr}\left(G \mid k(n)=k^{*}-1\right) & \geq-q \operatorname{Pr}\left(I \mid k(n)=k^{*}-1\right) .
\end{aligned}
$$

Hence $k^{*}(n)$ is implicitly defined by the following weak inequality:

$$
\left(\frac{p}{1-p}\right)^{2\left[k^{*}(n)-1\right]-n} \leq \frac{\pi}{1-\pi} \frac{q}{1-q} \leq\left(\frac{p}{1-p}\right)^{2 k^{*}(n)-n}
$$

The above defined voting rule ${ }^{8}$ is optimal in the sense that it induces the truthful revelation of signals, thus producing unanimously approved decisions given full information and fully updated posteriors. It is also efficient in the Bayesian hypothesis-testing sense, insofar as it minimizes posterior expected utility losses assuming a similar Bayesian loss function. Moreover, using the definition of $k^{*}(n)$ by Persico (2004), which he calls the statistical rule, i.e.,

$$
k^{*}(n)=\underset{n \in\{0,1, \ldots, N\}}{\operatorname{argmax}}[-q \pi \operatorname{Pr}(\text { Error } I ; n, k(n))-(1-q)(1-\pi) \operatorname{Pr}(\text { Error } I I ; n, k(n))],
$$

it is straightforward to argue that given informative voting the optimal aggregation rule minimizes the weighted sum of errors that forms the first part of the expected social welfare

\footnotetext{
${ }^{8}$ In order to make $k^{*}(n)$ well-defined and to rule out trivial cases, one may assume that parameters $(p, q, \pi)$ are such that $E[u(A, \cdot) \mid k(n)=0] \geq E[u(C, \cdot) \mid k(n)=0]$ and $E[u(C, \cdot) \mid k(n)=n] \geq E[u(A, \cdot) \mid k(n)=n]$, so that jurors may not always want to convict or acquit respectively.
} 
function in equation 1. One last crucial property of the $k^{*}(\cdot)$ function proven by Persico (2004) in lemma 1 is that $k^{*}(n+2)=k^{*}(n)+1$, assuming $k^{*}(n) \neq 0, n+1$. Essentially, $k^{*}(\cdot)$ behaves as a step function that increases by 1 as $n$ increases by $2 .^{9}$

Lemma 2 (Lemma 1 in Persico, 2004) Suppose $k^{*}(n) \neq 0, n+1$ for some $n=0,1, \ldots, N$. Then, $k^{*}(n+2)=k^{*}(n)+1$.

In light of the above definitions and assumptions of informative voting strategies, it is straightforward to calculate the two error probabilities by means of the binomial formula, i.e.,

$$
\begin{gathered}
\operatorname{Pr}\left(\text { Error } I ; n, k^{*}(n)\right)=\operatorname{Pr}\left(C \mid I ; n, k^{*}(n)\right)=\sum_{j=k^{*}(n)}^{n}\left(\begin{array}{c}
n \\
j
\end{array}\right)(1-p)^{j} p^{n-j} \\
\text { and } \\
\operatorname{Pr}\left(\text { Error } I I ; n, k^{*}(n)\right)=\operatorname{Pr}\left(A \mid G ; n, k^{*}(n)\right)=1-\sum_{j=k^{*}(n)}^{n}\left(\begin{array}{c}
n \\
j
\end{array}\right) p^{j}(1-p)^{n-j} .
\end{gathered}
$$

Hence, substituting in from equations 3 and 4 above, optimal jury size (equation 1) becomes

$$
n^{*}=\underset{0 \leq n \leq N}{\operatorname{argmax}}\left\{N\left[-q \pi \sum_{j=k^{*}(n)}^{n}\left(\begin{array}{c}
n \\
j
\end{array}\right)(1-p)^{j} p^{n-j}-(1-q)(1-\pi)\left(1-\sum_{j=k^{*}(n)}^{n}\left(\begin{array}{c}
n \\
j
\end{array}\right) p^{j}(1-p)^{n-j}\right)\right]-c n\right\}
$$

In essence, a social planner should increase the number of informative signals canvassed until the expected cost of added information exceeds the marginal social gains in the expected reduction of false convictions and acquittals. In its core, this is a theoretical question of optimal sample selection driven by the tension between the size-related transaction costs of deliberation and the positive statistical effects of wider representation.

In light of the discrete nature of the optimization problem, the analysis proceeds by deriving a set of necessary conditions for $n^{*}$ rather than a closed-form solution. First, let us

\footnotetext{
${ }^{9}$ Moreover, one can show that $\frac{k^{*}(n)}{n} \underset{n \rightarrow \infty}{\longrightarrow} \frac{1}{2}$, i.e., simple majority voting is optimal in the limit.
} 
define the marginal social benefit of adding one extra juror as

$$
\begin{aligned}
M B\left(n, k^{*}(n)\right) & =N\left\{-q \pi\left[\operatorname{Pr}\left(\text { Error } I ; n, k^{*}(n)\right)-\operatorname{Pr}\left(\text { Error } I ; n-1, k^{*}(n-1)\right)\right]\right. \\
& \left.-(1-q)(1-\pi)\left[\operatorname{Pr}\left(\text { Error } I I ; n, k^{*}(n)\right)-\operatorname{Pr}\left(\text { Error } I I ; n-1, k^{*}(n-1)\right)\right]\right\} \\
& =N\left\{-q \pi\left[\sum_{j=k^{*}(n)}^{n}\left(\begin{array}{c}
n \\
j
\end{array}\right)(1-p)^{j} p^{n-j}-\sum_{j=k^{*}(n-1)}^{n-1}\left(\begin{array}{c}
n-1 \\
j
\end{array}\right)(1-p)^{j} p^{n-1-j}\right]\right] \\
& \left.-(1-q)(1-\pi)\left[\sum_{j=k^{*}(n-1)}^{n-1}\left(\begin{array}{c}
n-1 \\
j
\end{array}\right) p^{j}(1-p)^{n-1-j}-\sum_{j=k^{*}(n)}^{n}\left(\begin{array}{c}
n \\
j
\end{array}\right) p^{j}(1-p)^{n-j}\right]\right\} .
\end{aligned}
$$

It should be noted that the marginal changes in error probabilities from adding one extra juror are not monotonically decreasing because of the shape of the $k^{*}(\cdot)$ step function. Whenever $k^{*}(n)=k^{*}(n-1)$, the Type $I$ error probability generally goes up (or stay the same), while the Type II error probability generally goes down, since only the upper bound of the summation increases (see equations 3 and 4). Intuitively, this happens because the same number of guilty signals needs to be canvassed by a larger pool of jurors. On the other hand, whenever $k^{*}(n)=k^{*}(n-1)+1$, the Type $I$ error probability generally decreases (or stays the same) and the Type II error probability generally increases, since adding an extra juror with an unchanged voting threshold increases the probability of conviction given informative voting and decreases the probability of acquittal. The opposite is the case when the expanded jury has to abide by a higher voting rule. The general trend of these error probabilities is decreasing as $n$ increases with the exception of some extreme parameter configurations. 
Following the above analysis, the local optimization condition boils down to

$$
\begin{gathered}
E W\left(n^{*}, k^{*}\left(n^{*}\right)\right) \geq E W\left(n^{*}+1, k^{*}\left(n^{*}+1\right)\right) \\
\Leftrightarrow A M B\left(n^{*}+1, k^{*}\left(n^{*}+1\right)\right) \leq \frac{c}{N}
\end{gathered}
$$

and

$$
\begin{gathered}
E W\left(n^{*}, k^{*}\left(n^{*}\right)\right) \geq E W\left(n^{*}-1, k^{*}\left(n^{*}-1\right)\right) \\
\Leftrightarrow A M B\left(n^{*}, k^{*}\left(n^{*}\right)\right) \geq \frac{c}{N}>0,
\end{gathered}
$$

where $A M B\left(n, k^{*}(n)\right)=\frac{M B\left(n, k^{*}(n)\right)}{N}$, which jointly imply the following necessary condition (local maximum)

$$
A M B\left(n^{*}, k^{*}\left(n^{*}\right)\right) \geq \frac{c}{N} \geq A M B\left(n^{*}+1, k^{*}\left(n^{*}+1\right)\right)
$$

Because of the step-like property of Type $I$ and $I I$ errors, the average marginal social benefit function $A M B(\cdot, \cdot)$ will generally have multiple local maxima, one of which will correspond to the globally optimizing committee design $\left(n^{*}, k^{*}\left(n^{*}\right)\right)$. All local maxima will either occur only at size levels $n$ where the voting threshold remains the same as in $n-1$ or only at points where there is a jump in $k^{*}(n)$. This would imply that there may not exist local optima at consecutive size levels $n$ and $n+1$. Whether local optima and, by consequence, the global optimum occur at a point where the optimal voting rule remains the same or increases will depend on the relative intensity of the opposite shifts in Type $I$ and $I I$ errors leading up to $n$ as determined by the parameter configuration $(q, \pi, p)$. Extreme values of the individual cost of participation $c$ relative to the total community population $N$ can generate some interesting cases. High enough cost values may lead to no committee formation $\left(n^{*}=0\right)$ depending on 
the parameter configuration $(q, \pi, p)$, in which case the defendant is either convicted when $k^{*}(0)=0$ or acquitted when $k^{*}(0)=1$. On the other hand, extremely low values of $c$ may possibly suggest direct democracy $\left(n^{*}=N\right)$ as an optimal decision-making mechanism, where universal participation is deemed socially optimal. ${ }^{10}$

The binomial formulae and the step-like $k^{*}(\cdot)$ function give rise to an average marginal social benefit function consisting of two branches of discrete points corresponding to the cases where $k^{*}(\cdot)$ either increases or stays the same as $n$ increases by one. One branch is always above the other in the sense that local optima may only located on the upper branch, which is represented by the sequence $\left\{A M B\left(n_{0}+2 k, k^{*}\left(n_{0}+2 k\right)\right)\right\}, k=0,1,2, \ldots\left(n_{0}\right.$ denoting the minimum jury size such that $\left.A M B\left(n_{0}, k^{*}\left(n_{0}\right)\right)>0\right)$. This implies that for all local optima $\widetilde{n} \in\{0,1, \ldots, N\}$ either $k^{*}(\widetilde{n})=k^{*}(\widetilde{n}-1)$ or $k^{*}(\widetilde{n})=k^{*}(\widetilde{n}-1)+1$. The range of the function almost always consists of non-negative numbers except for extreme cases of extremely high or low values of signal accuracy $p \in(.5,1]$. Its concavity is ambiguous within the relevant domain $\{0,1, \ldots, N\}$, since it is hard to disentangle the two opposing effects of an increase in committee size on the two types of errors. Asymptotically, $A M B\left(n, k^{*}(n)\right) \underset{n \rightarrow \infty}{\longrightarrow} 0$, i.e., both branches converge to the x-axis. ${ }^{11}$ Graph 1 below depicts $A M B(\cdot, \cdot), \frac{c}{N}$, and $n^{*}$ for different parameter configurations of $(q, \pi, p)$, where $N=100$ and $c=0.1$. The average social marginal benefit mappings always separate into two branches depending on the optimal voting rule $k^{*}(\cdot)$. The optimal jury size $n^{*}$ is depicted in the graph by a large dot and its corresponding $A M B\left(n^{*}, k^{*}\left(n^{*}\right)\right)$ by a dashed line. The horizontal lines parallel to the $\mathrm{x}$-axis represent the average marginal social cost lines $\frac{c}{N}$.

\footnotetext{
${ }^{10}$ In fact, some extreme parameter configuration, where $p$ is close to $1, \pi>\frac{1}{2}$, and $q<\frac{1}{2}$, give rise to the paradoxical situation of $n^{*}(N)<N$ even for $c=0$. That may occur because of the shape of the Error $I$ and $I I$ functions and the fact that the average social marginal benefit function may turn out negative for committee sizes $n$ close to the community size $N$.

${ }^{11}$ To prove this assertion, we need the fact mentioned in the footnote on page 9 that $\frac{k^{*}(n)}{n} \underset{n \rightarrow \infty}{\longrightarrow} \frac{1}{2}$. Then, combined with the assumption that $p \in\left(\frac{1}{2}, 1\right]$, the Law of Large Numbers and the Condorcet Jury Theorem imply that $\operatorname{Pr}\left(\right.$ Error $\left.I ; n, k^{*}(n)\right) \underset{n \rightarrow \infty}{\longrightarrow} 0$ and $\operatorname{Pr}\left(\right.$ Error $\left.I I ; n, k^{*}(n)\right) \underset{n \rightarrow \infty}{\longrightarrow} 0$, which in turn proves our claim.
} 

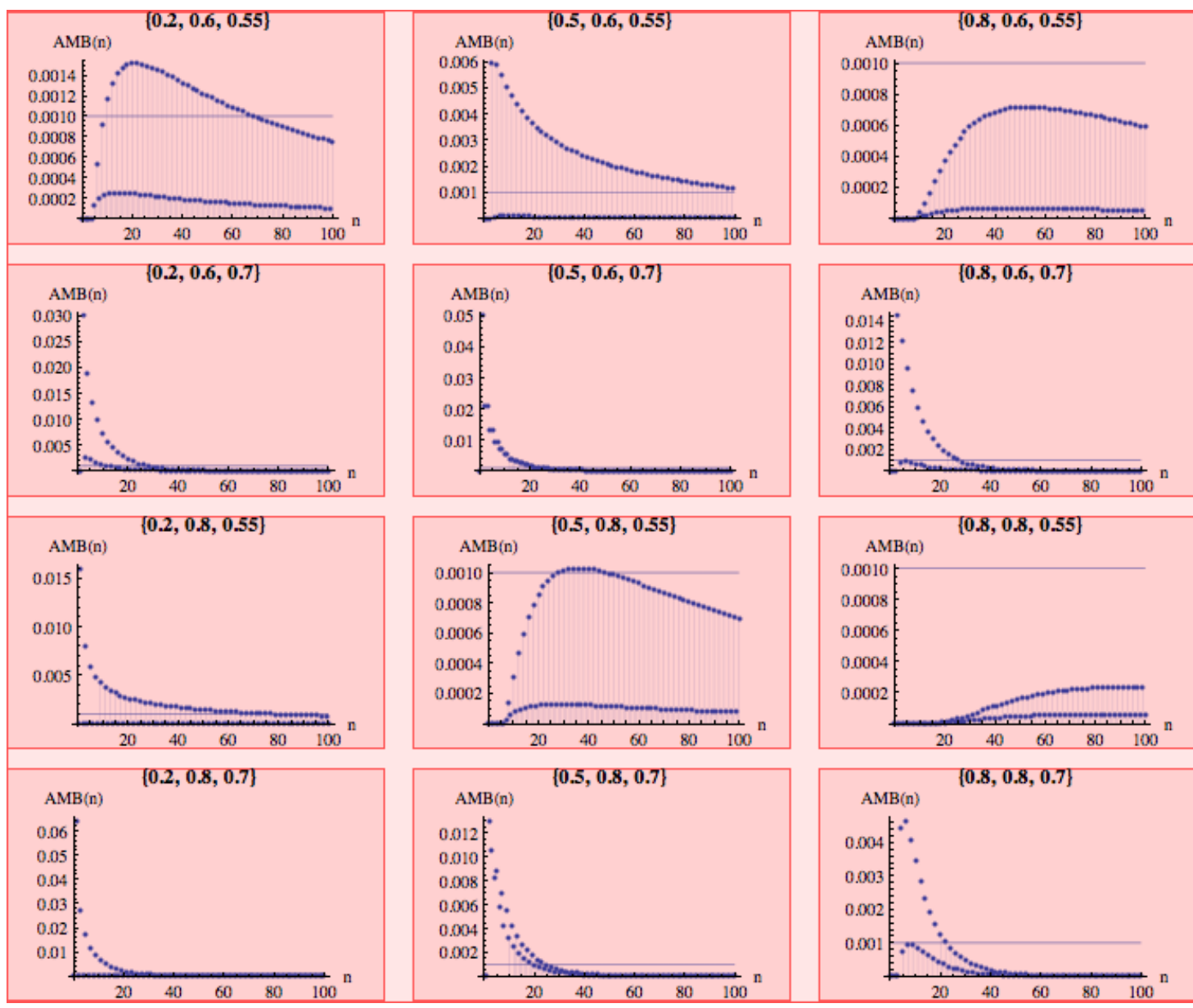

Figure 1: Average social marginal benefit mappings 
Ultimately, globally optimal $n^{*}$ is determined by the average marginal social cost line $\frac{c}{N}$. Since it must be a local maximum as well, it must lie on the upper branch of the $A M B(\cdot, \cdot)$ function. Assuming is strictly positive, it must be at a point where (i) the upper branch approaches $\frac{c}{N}$ from above, (ii) it is a local optimum satisfying condition 7, and (iii) maximizes expected social welfare among all such points that satisfy necessary conditions (i) and (ii). Let $n_{+}^{*}$ denote such a strictly positive jury size that satisfies these conditions. Then, $n^{*}=\underset{\operatorname{argmax}}{E W}(\cdot, \cdot)$. In light of the discrete nature of this optimization problem, $n \in\left\{0, n_{+}^{*}\right\}$

the solution will be generically unique.

At this point it seems pertinent to discuss the global optimality of the $\left(n^{*}, k^{*}\left(n^{*}\right)\right)$ solution to the dual mechanism design problem of optimal committee size and voting rule. Having restricted the attention to informative voting equilibria in this finite environment bounded by the size $N$ of the community, the question arises whether the existence of responsive or non-responsive, monotonic or non-monotonic, mixed-strategy voting equilibria à la Feddersen and Pesendorfer (1998) would nullify the global social optimality of the above solution. Clearly, $\left(n^{*}, k^{*}\left(n^{*}\right)\right)$ is a local optimum, since as shown above $n^{*}$ is optimal given $k^{*}(\cdot)$ and by definition $k^{*}\left(n^{*}\right)$ is the optimal aggregation rule for any given jury size. It turns out that it is also a global optimum by dint of the powerful properties of the optimal voting rule.

Claim 1 Social expected welfare of a community of size $N$ is globally maximized at $\left(n^{*}, k^{*}\left(n^{*}\right)\right)$.

The intuition behind this claim becomes clear by looking at the problem from the angle of the mechanism designer or social planner, whose basic task is to induce perfect information aggregation at a minimal cost for the community. Remember that $k^{*}(\cdot)$ is the only aggregation rule that will induce the rational full revelation of jurors' private information. As argued above and in Persico (2004), the statistical rule $k^{*}(n)$ entails the ex ante minimization of the weighted sum of errors (or else the maximization of the social benefit part of the expected welfare function in equation 1). Hence, in anticipation of this full revelation of signals, 
the mechanism designer will choose the optimal jury size $n^{*}$ so as to balance the ex ante information aggregation benefits (which are bounded above zero for any finite $n$ ) with the social costs of jury draft. Given the definition of $n^{*}$ and $k^{*}(\cdot)$, the solution $\left(n^{*}, k^{*}\left(n^{*}\right)\right)$ is then the global social optimum among local social optima $\left(\widetilde{n}, k^{*}(\widetilde{n})\right.$ ) (see figure 2 where bold dots denote local social optima and the circled dot denotes the global social optimum). Allowing for alternative responsive, non-monotonic, and/or asymmetric equilibria would certainly not enhance the information aggregation component of expected social welfare. Thus, it would only be possible to increase social welfare by cutting back on jury size. However, following Feddersen and Pesendorfer's (1998) derivation of the error probabilities of non-unanimous voting rules, the positive effect of economizing on voting costs by curtailing jury size is dominated by the loss in information aggregation precision.

\subsection{Comparative Statics}

One can now perform some exercises in comparative statics by determining the effects of parameter changes on optimal jury size. The following proposition summarizes the results:

Proposition 1 Optimal jury size is ceteris paribus (i) generally weakly increasing up to some $p_{1}$, constant for any $p \in\left[p_{1}, p_{2}\right]$, where $p_{1}<p_{2}$, and weakly decreasing for $p \in\left(p_{2}, 1\right]$, (ii) unambiguously weakly decreasing with respect to the voting cost c, and (iii) unambiguously weakly increasing in community size $N$. Changes in parameters $\pi$ and $q$ have an ambiguous effect on $n^{*}$.

First, let us consider the effects of an increase in signal accuracy $p$. Initially, the insufficient accuracy of extremely low values of $p$ does not justify the participation costs of forming any kind of committee; instead, the community is content to rely on its prior information. Subsequently, increasing values of $p$ raise the average marginal social benefit of participation in relation to the average marginal social cost so that $n^{*}$ becomes positive and weakly increasing 


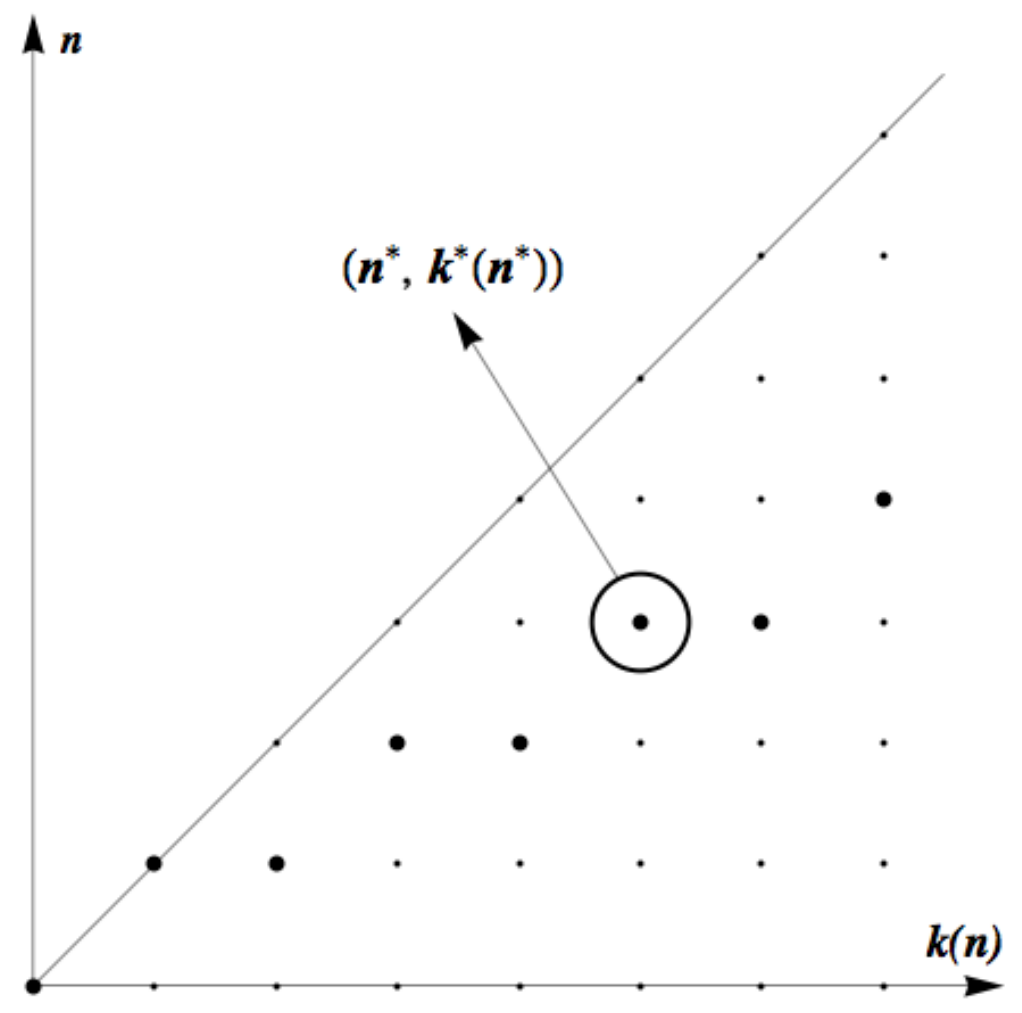

Figure 2: Locally and globally optimal combinations of committee size and voting rule 
up to some $p_{1}$, at which point it plateaus at its maximum value up to $p_{2}$. For increasingly high values of $p>p_{2}$, the $A M B(\cdot, \cdot)$ function becomes convex and convergent to zero (see cases with $p=0.7$ in figure 1 ), so that the information gain relative to the participation cost justifies only weakly decreasing optimal committee sizes. The intuition is quite simple: for increasing levels of signal informativeness (or in other words expertise), one need canvass fewer and more accurate signals in order to achieve the socially optimal level of errors. Some spikes that may appear to contradict the general pattern of the relationship between $n^{*}$ and $p$ are due to threshold effects caused by jumps in the optimal voting rule function $k^{*}(\cdot)$. Figure 3 below illustrates the relationship through some numerical examples with $N=100, c=0.1, q \in\{0.1,0.5,0.9\}$, and $\pi \in\{0.6,0.8\}$. With the exception of some upward and downward spikes due to the discontinuities of the voting rule, the general pattern is one of single-peakedness.
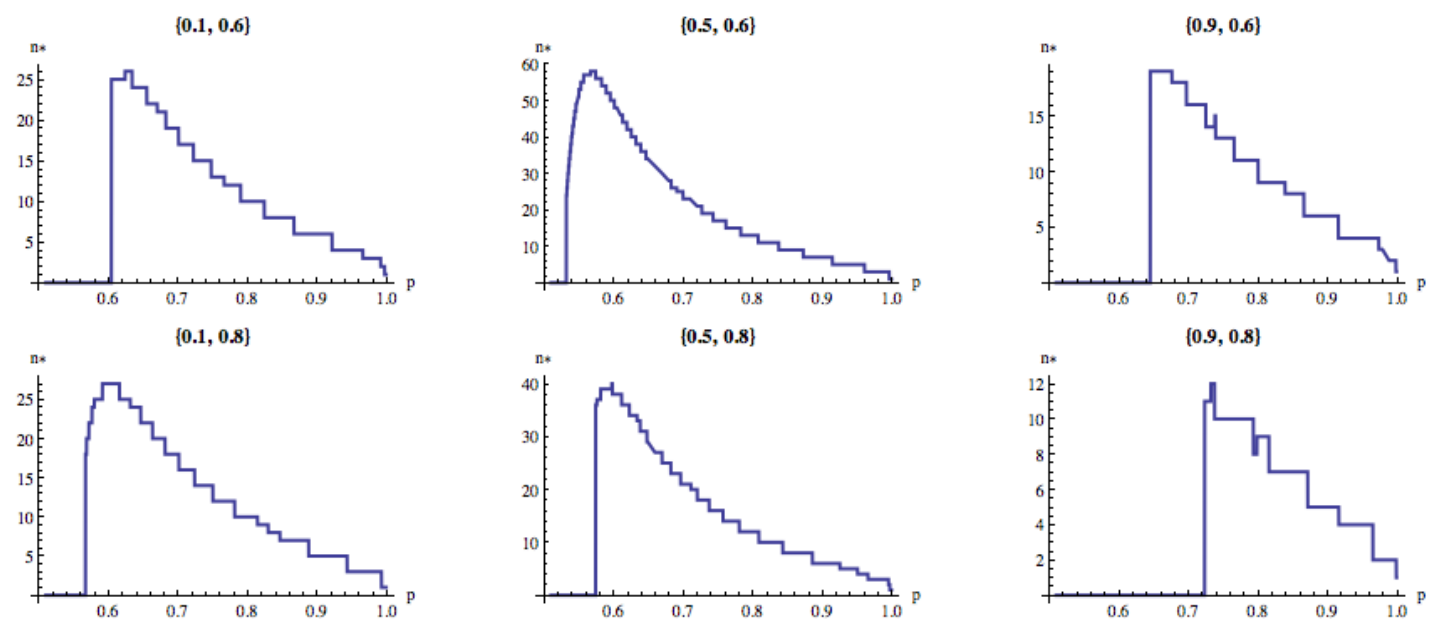

Figure 3: Relationship between signal accuracy $p$ and optimal committee size $n^{*}$

The effect of parameters $\pi$ and $q$ follows the same pattern within the overlapping parameter range of $\left[\frac{1}{2}, 1\right)$, since they enter symmetrically into both the average marginal social benefit and optimal voting rule functions. Even though an unambiguous general pattern may not 
be established, given the threshold effects of discontinuous changes in $k^{*}(\cdot)$, it appears that there is a weakly decreasing trend in their effect on the optimal size of a committee. One can intuitively argue that the more certain and valuable is the innocence of the defendant, the smaller the socially optimal size of a jury designated to adjudicate the case. In light of strong informational and preference biases, the community becomes less willing to expend political resources in search of the correct decision. Correspondingly, for values of $q \in\left[0, \frac{1}{2}\right)$, the relatively higher utility loss associated with an erroneous acquittal generally give rise to a weakly increasing trend in the relationship between $q$ and $n^{*}$.

Finally, an exogenous decrease in $c$ or an increase in $N$ may only increase optimal jury size $n^{*}$. For any $c>c^{\prime}>0$ or $N^{\prime}>N>0$, the average marginal social cost lines $\frac{c^{\prime}}{N}$ or $\frac{c}{N^{\prime}}$ shift downwards in a parallel fashion, thus rendering $n^{*}(c ; N)$ suboptimal and accordingly increasing optimal jury size to $n^{*}+2 k$ for some positive integer $k$. To prove this, let $n^{*}(N)=$ $n^{*}$, which implies that for all $n^{\prime}<n^{*}$,

$$
\begin{aligned}
E W\left(n^{*}, k^{*}\left(n^{*}\right) ; N\right) & >E W\left(n^{\prime}, k^{*}\left(n^{\prime}\right) ; N\right) \\
E W\left(0, k^{*}(0) ; N\right)+\sum_{n=1}^{n^{*}} M B\left(n, k^{*}(n) ; N\right)-n^{*} c & >E W\left(0, k^{*}(0) ; N\right)+\sum_{n=1}^{n^{\prime}} M B\left(n, k^{*}(n) ; N\right)-n^{\prime} c \\
N \sum_{n=n^{\prime}+1}^{n^{*}} A M B\left(n, k^{*}(n)\right) & >\left(n^{*}-n^{\prime}\right) c .
\end{aligned}
$$

Since this will continue to hold for any $N^{\prime}>N$, then $n^{*}\left(N^{\prime}\right) \geq n^{*}(N)$, i.e., optimal committee size is weakly increasing in community size $N$ (and by the same argument weakly decreasing in $c$ ).

In examining the asymptotic properties of the model, I find that $n^{*}$ converges asymptotically to a finite limit. This is certainly quite plausible; for very large communities the overall benefits of better information aggregation and accuracy of verdict that come with increases in 
committee size would eventually peter out relative to the constant technology drafting costs $c$. In that sense, there is an upper bound on $n^{*}$ and the asymptotic ratio $\lim _{N \rightarrow \infty} \frac{n^{*}(N)}{N}$ equals zero since in the limit the linear social costs of voting will dwarf the plateauing social benefits of increased participation. ${ }^{12}$ Clearly, by the Law of Large Numbers and the Condorcet Jury Theorem, a growing sample of signals canvassed from a large number of people will almost surely reveal the true state of the world, thus eliminating the utility costs associated with wrong decisions. Hence, it is asymptotically inefficient to increase jury size in direct proportion to the overall size of the community, which leads us to the following proposition:

Proposition 2 For all $q \in[0,1], \pi \in\left[\frac{1}{2}, 1\right)$, and $p \in\left(\frac{1}{2}, 1\right]$ such that $k^{*}(\cdot)$ is well-defined, then $n^{*}(N) \underset{N \rightarrow \infty}{\longrightarrow} n_{\bar{l}}<\infty$, i.e., optimal jury size converges to a finite upper bound as the actual size of the community grows infinitely.

Proof. See appendix.

One interesting implication of the above analysis is that smaller communities or communities with higher costs of drafting or voting (for example, because of higher mobility costs, bureaucratic frills, and/or poor levels of institutionalization) are all else equal more likely to be optimally governed by more 'oligarchic' and concentrated decision-making institutions. This result has a paradoxical flavor to it and runs counter to the Aristotelean or even Rousseauean normative tenet that smaller countries are better suited for direct democracy, whereas larger countries should be autocratically governed.

\subsection{Nash Implementability}

What if instead of a draft the mechanism designer allowed for voluntary participation in communal decision-making, namely through self-selection into a jury? Would the members of

\footnotetext{
${ }^{12}$ Taking national legislative bodies as proxies for decision-making committees, then the commonplace observation that the size of legislatures falls way short of direct proportionality with a country's population size would confirm the above intuition.
} 
the community rationally achieve optimal levels of participation? To answer these questions, the rest of the paper examines how the expected level of Nash participation compares to the optimal jury size level $n^{*}$ for a fixed voting rule $k^{*}(\cdot)$.

The first step is to solve the two-stage game where $N$ members of a community initially decide whether to participate in a jury for a given cost $c$ and subsequently how to vote based on their privately received information. The defendant is then convicted as long as $k^{*}(n)$ or more of the voluntary jury members vote to convict and is acquitted otherwise. I, therefore, implicitly assume that the mechanism designer can make a credible commitment to enforcing the optimal voting rule upon observing participation decisions. This remains a common values game with utilities as specified above. The set-up of the game is typical of voting games of abstention with positive voting costs, yet also highlights the public goods nature of informed communal decision-making. It turns out that public participation in communal decision-making fora is socially suboptimal because citizens fail to internalize the social benefits of their individual participation.

Let the participation strategy denoted by $\tau(\cdot)$ be a function of individual participation $\operatorname{costs} c$. The analysis will focus on symmetric equilibria, whereby individual signals do not affect participation decisions. ${ }^{13}$ Effectively, the emphasis is on making the right decision irrespective of the relative costs of different types of errors. Pure strategy Nash equilibria will typically not exist as is the case in the voter abstention literature (see for example Palfrey and Rosenthal, 1983, 1985) except under special circumstances (e.g., when no jury formation is optimal, then $\left(\tau_{i}^{*}=0\right)_{i=1, \ldots, N}$ is a Nash equilibrium strategy profile). The existence, however, of a symmetric mixed strategy Nash equilibrium is guaranteed by fixed-point theorems.

In order to derive equilibrium participation in this game, ${ }^{14}$ we need to determine the $e x$

\footnotetext{
${ }^{13}$ Supposing that agents only receive their private information upon committing to participate or not, the emphasis on symmetric equilibria appears natural. Accordingly neither type of information (either in favor of guilt or innocence) will induce any of the players to renege upon their commitment.

${ }^{14}$ The analysis is an extension of the approach in Ghosal and Lockwood (2003) and Börgers (2004).
} 
ante expected marginal gain of participation $B(\cdot)$ for agent $i$, given that everyone expects an informative voting equilibrium in the voting endgame. Let $\tau$ denote the ex ante probability of participation by all members of the population. Then

$$
\begin{aligned}
B(\tau) & =\sum_{n=0}^{N-1} m(n ; \tau) \operatorname{Pr}(\text { piv; } n) b(n) \\
& =\sum_{n=0}^{N-1}\left(\begin{array}{c}
N-1 \\
n
\end{array}\right) \tau^{n}(1-\tau)^{N-1-n}\left(\begin{array}{c}
n \\
k^{*}(n+1)-1
\end{array}\right)(\operatorname{Pr}(g))^{k^{*}(n+1)-1}(\operatorname{Pr}(i))^{n-k^{*}(n+1)+1} b(n),
\end{aligned}
$$

where $m(n ; \tau)$ denotes the probability that $n$ out of all agents other than $i$ choose to participate given symmetric participation probability $\tau$ and $\operatorname{Pr}($ piv;n) denotes the probability that $i$ 's vote would be pivotal, given that $n$ other jurors have chosen to participate (i.e., that he/she would be pivotal in a jury of size $n+1) . \operatorname{Pr}(g)$ is the ex ante unconditional probability that $j \neq i$ receives a guilty signal and is equal to $\pi(1-p)+(1-\pi) p$. Same for $\operatorname{Pr}(i)=\pi p+(1-\pi)(1-p)$. Lastly, $b(n)$ denotes the ex ante marginal gain from participation given that $i$ is pivotal in a jury of size $n+1$.

Since in a fully informative voting equilibrium it is a best response for $i$ to vote his or her signal if pivotal (from equation 2 and the definition of $k^{*}$ ), then it follows that for 
$k^{*}(n+1)=k^{*}(n)$

$$
\begin{aligned}
\widehat{b}(n) & =-q \operatorname{Pr}(g) \operatorname{Pr}\left(I \mid k(n+1)=k^{*}(n+1)\right)-(1-q) \operatorname{Pr}(i) \operatorname{Pr}\left(G \mid k(n+1)=k^{*}(n+1)-1\right) \\
& +(1-q) \operatorname{Pr}\left(G \mid k(n)=k^{*}(n)-1\right) \\
& =-q \operatorname{Pr}(g) \frac{\pi\left(\begin{array}{c}
n+1 \\
k^{*}(n+1)
\end{array}\right)(1-p)^{k^{*}(n+1)} p^{n+1-k^{*}(n+1)}}{\left(\begin{array}{c}
n+1 \\
k^{*}(n+1)
\end{array}\right)(\operatorname{Pr}(g))^{k^{*}(n+1)}(\operatorname{Pr}(i))^{n+1-k^{*}(n+1)}} \\
& -(1-q) \operatorname{Pr}(i) \frac{(1-\pi)\left(\begin{array}{c}
n+1 \\
k^{*}(n+1)
\end{array}\right) p^{k^{*}(n+1)-1}(1-p)^{n+2-k^{*}(n+1)}}{\left(\begin{array}{c}
n+1 \\
k^{*}(n+1)
\end{array}\right)(\operatorname{Pr}(g))^{k^{*}(n+1)-1}(\operatorname{Pr}(i))^{n+2-k^{*}(n+1)}} \\
& +(1-q) \frac{(1-\pi)\left(\begin{array}{c}
n \\
k^{*}(n+1)-1
\end{array}\right) p^{k^{*}(n+1)-1}(1-p)^{n+1-k^{*}(n+1)}}{\left(\begin{array}{c}
n \\
k^{*}(n+1)-1
\end{array}\right)(\operatorname{Pr}(g))^{k^{*}(n+1)-1}(\operatorname{Pr}(i))^{n+1-k^{*}(n+1)}} \\
& =\ldots=\frac{[p(1-p)]^{k^{*}(n)}\left[(1-q)(1-\pi)(1-p)^{n+1-2 k^{*}(n)}-q \pi p^{n+1-2 k^{*}(n)}\right]}{[\pi(1-p)+(1-\pi) p]^{k^{*}(n)-1}[\pi p+(1-\pi)(1-p)]^{n+1-k^{*}(n)}}
\end{aligned}
$$

while similarly for $k^{*}(n+1)=k^{*}(n)+1$,

$$
\begin{aligned}
\widetilde{b}(n) & =-q \operatorname{Pr}(g) \operatorname{Pr}\left(I \mid k(n+1)=k^{*}(n+1)\right)-(1-q) \operatorname{Pr}(i) \operatorname{Pr}\left(G \mid k(n+1)=k^{*}(n+1)-1\right) \\
& +q \operatorname{Pr}\left(I \mid k(n)=k^{*}(n)\right) \\
& =\ldots=\frac{[p(1-p)]^{k^{*}(n)}\left[q \pi p^{n+1-2 k^{*}(n)}-(1-q)(1-\pi)(1-p)^{n+1-2 k^{*}(n)}\right]}{[\pi(1-p)+(1-\pi) p]^{k^{*}(n)}[\pi p+(1-\pi)(1-p)]^{n-k^{*}(n)}}
\end{aligned}
$$

The only difference between the above two expressions is what happens when $i$ decides not to participate. In the first case (equation 9) the defendant will be acquitted by a jury of size $n$ (since the number of convicting votes would be one short of the threshold) and in the second case (equation 10) the defendant will be convicted by a jury of the same size (since the voting threshold would decrease by one). Note that both expressions turn out to be weakly positive given the implicit definition of $k^{*}(\cdot)$ in inequality 2 . 
Hence, $B(\tau)$ becomes

$$
\begin{aligned}
B(\tau) & =\sum_{n=0}^{N-1} m(n ; \tau) \operatorname{Pr}\left(\text { piv;n) }\left[\mathbf{1}\left(k^{*}(n+1)=k^{*}(n)\right) \widehat{b}(n)+\mathbf{1}\left(k^{*}(n+1)=k^{*}(n)+1\right) \widetilde{b}(n)\right]\right. \\
& =\sum_{n=0}^{N-1}\left(\begin{array}{c}
N-1 \\
n
\end{array}\right) \tau^{n}(1-\tau)^{N-1-n}[p(1-p)]^{k^{*}(n)}\left(\begin{array}{c}
n \\
k^{*}(n)-\mathbf{1}\left(k^{*}(n+1)=k^{*}(n)\right)
\end{array}\right) \\
& \times\left|(1-q)(1-\pi)(1-p)^{n+1-2 k^{*}(n)}-q \pi p^{n+1-2 k^{*}(n)}\right|
\end{aligned}
$$

where $\mathbf{1}(\cdot)$ is the indicator function of two exhaustive and mutually exclusive events. Obviously the fact that $B(\tau)$ is a binomial expansion weighted by a sequence of positive and increasing factors makes it non-monotonic, therefore not amenable to the uniqueness of a fixed point.

The mixed-strategy Nash equilibrium occurs at $B\left(\tau^{*}\right)=c$, i.e., when the symmetric mixed participation strategy of all $j \neq i$ makes $i$ ex ante indifferent between participating in the jury or not. Pure strategies $\tau \in\{0,1\}$ may still arise in equilibrium as corner solutions whenever $c$ is too high or too low. Moreover multiple low- and high- participation equilibria are possible because of the non-monotonicity of the ex ante expected marginal gain of participation function $B(\cdot) .15$

The next step in the analysis would be to compare the expected jury size $E[n]=\tau^{*} N$ under the above mixed strategy equilibria and the optimal jury size $n^{*}$ derived from the previous section. The following proposition states the results:

Proposition 3 For all $q \in[0,1], \pi \in\left[\frac{1}{2}, 1\right)$, and $p \in\left(\frac{1}{2}, 1\right]$ such that $k^{*}(\cdot)$ is well-defined, the maximal element of the symmetric mixed strategy equilibrium set $\max \tau^{*}$ is lower or equal to the socially optimal level $\tau^{\text {opt }}=\frac{n^{*}}{N}$, i.e., voluntary participation in communal decision-

\footnotetext{
${ }^{15}$ One of course cannot exclude the existence of asymmetric equilibria, whereby each player conditions his/ her participation strategy on his/her interim information type and, thus, uses Bayesian updating to determine the probability of being pivotal.
} 
making in an environment with common values will be suboptimally low.

Proof. First, define $\tau^{o p t}$ such that $\tau^{o p t} N=n^{*}$ and assume only interior equilibrium mixed strategies $\tau^{*} \in(0,1)$. It only needs to be proven that for each $\tau^{*}$ it is ex ante weakly Paretoimproving to increase $\tau$ and, hence, raise expected participation levels $\tau^{*} N$ up to $n^{*}$. This would imply that $\max \tau^{*} \leq \tau^{o p t}=\frac{n^{*}}{N}$.

The symmetric ex ante unconditional (on the signal $s_{i}$ ) utility of a citizen $i$ with common interior participation probability $\tau$ is

$$
U(\tau)=(1-\tau) u_{a}(\tau)+\tau\left(u_{p}(\tau)-c\right)
$$

where $u_{a}(\tau)$ is the ex ante unconditional utility of abstention

$$
\begin{gathered}
u_{a}(\tau)=\sum_{n=0}^{N-1}\left(\begin{array}{c}
N-1 \\
n
\end{array}\right) \tau^{n}(1-\tau)^{N-1-n} \\
\times\left[-q \pi \sum_{j=k^{*}(n)}^{n}\left(\begin{array}{c}
n \\
j
\end{array}\right)(1-p)^{j} p^{n-j}-(1-q)(1-\pi)\left(1-\sum_{j=k^{*}(n)}^{n}\left(\begin{array}{c}
n \\
j
\end{array}\right) p^{j}(1-p)^{n-j}\right)\right]
\end{gathered}
$$

and $u_{p}(\tau)$ is the ex ante unconditional utility of participation

$$
\begin{gathered}
u_{p}(\tau)=\sum_{n=0}^{N-1}\left(\begin{array}{c}
N-1 \\
n
\end{array}\right) \tau^{n}(1-\tau)^{N-1-n} \\
\times\left[-q \pi \sum_{j=k^{*}(n+1)}^{n+1}\left(\begin{array}{c}
n+1 \\
j
\end{array}\right)(1-p)^{j} p^{n+1-j}-(1-q)(1-\pi)\left(1-\sum_{j=k^{*}(n+1)}^{n+1}\left(\begin{array}{c}
n+1 \\
j
\end{array}\right) p^{j}(1-p)^{n+1-j}\right)\right] .
\end{gathered}
$$

Note that both these unconditional utilities are positively increasing in $\tau$, i.e., $u_{a}^{\prime}(\tau)>0$ and $u_{p}^{\prime}(\tau)>0$, since they are both summations of converging negative terms weighted by a binomial expansion. As such, an increase in $\tau$ leads to the first-order stochastic dominance 
of the binomial expansion with parameters $\tau \prime(>\tau)$ and $N-1 .^{16}$

Finally, let us take the first derivative of $U(\tau)$ in equation 12 substituting in for equilibrium values of $\tau$ :

$$
\begin{aligned}
U^{\prime}\left(\tau^{*}\right) & =\left(1-\tau^{*}\right) u_{a}^{\prime}(\tau)+\tau^{*} u_{p}^{\prime}\left(\tau^{*}\right)+u_{p}\left(\tau^{*}\right)-u_{a}\left(\tau^{*}\right)-c \\
& =\left(1-\tau^{*}\right) u_{a}^{\prime}(\tau)+\tau^{*} u_{p}^{\prime}\left(\tau^{*}\right)+B\left(\tau^{*}\right)-c \\
& =\left(1-\tau^{*}\right) u_{a}^{\prime}\left(\tau^{*}\right)+\tau^{*} u_{p}^{\prime}\left(\tau^{*}\right) \geq 0 .
\end{aligned}
$$

The last step follows from the above equilibrium condition $B\left(\tau^{*}\right)=c$. Hence, for each equilibrium participation probability $\tau^{*}$ it is weakly Pareto-improving to raise it. Since this applies to the maximal element of the equilibrium set of symmetric mixed strategies, then $\max \tau^{*} \leq \tau^{\text {opt }}=\frac{n^{*}(N)}{N}$. Note that if degenerate pure strategy equilibria exist, i.e., if $\tau^{*} \in\{0,1\}$, then it can be shown that $n^{*}=0$ or $N$ respectively, so that the proposition holds for boundary equilibria as well. Q.E.D.

Since it has been demonstrated that the equilibrium probability of participation is bounded above by $\frac{n^{*}(N)}{N}$ and the asymptotics of that limit have been characterized in proposition 2 , then it can be shown that symmetric mixed strategy equilibria converge to zero participation probabilities. This result runs parallel to the findings of Palfrey and Rosenthal (1985) in their analysis of asymptotic voting turnout equilibria under full information.

Moreover, as $N$ becomes large and $\tau$ decreases asymptotically, the binomial expression in $B(\tau)$ can be approximated by the Poisson distribution (for large $N$ and low $\tau$ ), i.e., $\sum_{n=0}^{N-1}\left(\begin{array}{c}N-1 \\ n\end{array}\right) \tau^{n}(1-\tau)^{N-1-n} \approx \sum_{n=0}^{\infty} \frac{e^{-(N-1) \tau}[(N-1) \tau]^{n}}{n !}$. In fact, Myerson (1998a,b) shows that the Poisson distribution would be exact if and only if the size of the community were a Poisson random variable with mean $N$. He further makes use of an approximation formula for pivot probabilities to show that the low-turnout equilibrium solution in Palfrey and

\footnotetext{
${ }^{16}$ See for example proposition 3 in Ghosal and Lockwood (2003).
} 
Rosenthal (1985) is unique in the limit. An appropriate modification of large game Poisson formulae for non-majoritarian voting rules would arguably prove uniqueness of the generic low-participation asymptotic equilibrium in this setting too.

\section{Bayesian Environment}

This section extends the basic framework of the model into a Bayesian environment with heterogeneous private costs. Let nature assign private participation $\operatorname{costs} c_{i}$ distributed according to a regular distribution function $F[\underline{c}, \bar{c}]$, where $F^{\prime}(\cdot)>0$ within the support $[\underline{c}, \bar{c}]$ (full support assumption). Thus, each agent $i$ 's private type is defined by $\left(c_{i}, s_{i}\right)$. The game structure is as follows: first, nature determines the unknown state of the world $I$ or $G$ and also assigns private types $\left(c_{i}, s_{i}\right)$ to each citizen $i$ in the community $N$. Then, the agents decide whether to become jurors or not. Finally, jurors vote either to convict or acquit based on their private signals and the optimal voting rule $k^{*}(n)$.

One can now solve for the symmetric Bayesian equilibrium of this two-stage voting game based once again on the simplifying assumption of informative voting, i.e., $\sigma(i)=0$ and $\sigma(g)=1$, in the final voting stage, which given $k^{*}(n)$ constitutes a Nash equilibrium in that subgame. Let $\tau:[\underline{c}, \bar{c}] \rightarrow[0,1]$ denote the symmetric participation strategy in the first stage of the game. It follows in a similar fashion to the above analysis that citizen $i$ will decide to participate in the decision-making process if and only if $B_{i}(\tau) \geq c_{i}$, otherwise he/she will decide to abstain. What follows is a proof of the existence of a symmetric Bayesian equilibrium in this generalized setting: ${ }^{17}$

Proposition 4 There exists at least one symmetric Bayesian equilibrium defined by an equilibrium cutoff $c^{*}$ whereby $\tau^{*}\left(c_{i}\right)=1$ if and only if $c_{i}<c^{*}$, otherwise $\tau^{*}\left(c_{i}\right)=0$. If there

\footnotetext{
${ }^{17}$ See proposition 1 in Ghosal and Lockwood (2003) for an existence proof in the case of majority voting.
} 
exists a fixed point such that $B\left(F\left(c^{*}\right)\right)=c^{*}$, then $c^{*}$ is a symmetric equilibrium cutoff point. If $B(1) \geq \bar{c}$, then $\bar{c}$ is an equilibrium cutoff. If for all $n \in\{0,1, \ldots, N\}$,

$$
[p(1-p)]^{k^{*}(n)}\left(\begin{array}{c}
n \\
k^{*}(n+1)-1
\end{array}\right)\left|q \pi p^{n+1-2 k^{*}(n)}-(1-q)(1-\pi)(1-p)^{n+1-2 k^{*}(n)}\right| \leq \underline{c},
$$

then $\underline{c}$ is the unique equilibrium cutoff.

A symmetric Bayesian equilibrium occurs when all $i$ 's have the same participation strategy $\tau^{*}$. One can define an equilibrium cutoff point such that the ex ante equilibrium probability of participation $\tau$ equals $F\left(c^{*}\right)$. The continuity of $B \circ F$ within the support $[\underline{c}, \bar{c}]$ guarantees the existence of an equilibrium cutoff either as a fixed point $B\left(F\left(c^{*}\right)\right)=c^{*}$ or as a boundary point of the support function. Note that the non-monotonicity of the $B \circ F$ function allows for multiple equilibria. ${ }^{18}$ Moreover, if the expected marginal gain from participation for a given number of other jury participants never goes above the lower cost bound $\underline{c}$, then no one will ever choose to participate in communal decision-making in such an equilibrium and $\underline{c}$ becomes the unique equilibrium cutoff point.

It must be noted that the focus has been restricted to the general class of symmetric Bayesian Nash equilibria assuming informative voting in the endgame. Obviously, allowing for weakly dominated strategies in the voting game gives rise to a number of other uninteresting and non-focal equilibria (for example where all vote to convict or acquit regardless of their signals) that alter the strategic calculus of participation.

\subsection{Social Efficiency and Mechanism Design}

Based on the analysis in subsection 2.1 one can now derive the socially optimal cutoff point $c^{\text {opt }}$ for the above Bayesian set-up. Let $c:[\underline{c}, \bar{c}] \rightarrow\{0,1\}$ denote any monotonic cutoff mechanism

\footnotetext{
${ }^{18}$ Börgers (2004) proves uniqueness of the symmetric Bayesian equilibrium in the case of private values.
} 
such that $\tau_{i}=1 \Leftrightarrow c_{i}<c$ and $\tau_{i}=0 \Leftrightarrow c_{i} \geq c$, i.e., citizens are drafted for jury (or not) depending on whether their reported cost types are above (or below) a certain threshold value. A social planner will want to implement such a mechanism that maximizes ex ante social welfare, that is before learning about realized individual costs. The ex ante efficient and incentive-compatible mechanism in this case is any optimal solution to the following optimization problem:

$$
\begin{aligned}
c^{\text {opt }} & =\underset{c \in[\underline{c}, \bar{c}]}{\operatorname{argmax}} \sum_{n=0}^{N}\left(\begin{array}{l}
N \\
n
\end{array}\right)[F(c)]^{n}[1-F(c)]^{N-n} \sum_{i=1}^{N} u_{i}(n, c(\cdot)) \\
& =\underset{c \in[\underline{c}, \bar{c}]}{\operatorname{argmax}} \sum_{n=0}^{N}\left(\begin{array}{l}
N \\
n
\end{array}\right)[F(c)]^{n}[1-F(c)]^{N-n} \\
& \times\left\{N\left[-q \pi \operatorname{Pr}\left(\text { Error I; } n, k^{*}(n)\right)-(1-q)(1-\pi) \operatorname{Pr}\left(\operatorname{Error} I I ; n, k^{*}(n)\right)\right]-n \int_{\underline{c}}^{c} c_{i} d F\left(c_{i}\right)\right\} .
\end{aligned}
$$

It should be noted that the above optimal mechanism places equal weight to all members of the community as a reflection of the 'veil of ignorance' symmetry of the game. That being said, $c^{\text {opt }}(\cdot)$ does not exhaust the full set of ex ante efficient mechanisms. ${ }^{19}$

It has, thus, been shown that $c^{o p t}$ is the ex ante socially optimal cutoff point in the Bayesian game from the perspective of the entire community. However, the ex ante efficient mechanism of political participation is not implementable by the Bayesian Nash equilibrium of the game. To validate this claim, it would suffice to show that max $c^{*} \leq c^{o p t}$.

Proposition 5 For all $q \in[0,1], \pi \in\left[\frac{1}{2}, 1\right)$, and $p \in\left(\frac{1}{2}, 1\right]$ such that $k^{*}(\cdot)$ is well-defined, the maximal symmetric equilibrium $\max c^{*}$ is lower or equal to the socially optimal cutoff point $c^{\text {opt }}$.

\footnotetext{
${ }^{19}$ Moreover, as noted in Myerson (1997), the set of ex ante efficient mechanisms will be a subset of interim efficient mechanisms (i.e., for realized private cost types), which in turn are a subset of ex post efficient mechanisms (i.e., given full information about cost types).
} 
Proof. For interior equilibrium cutoff points $c^{*} \in(\underline{c}, \bar{c})$ I only need to prove that for each $c^{*}$ it is ex ante Pareto-improving to increase the cutoff cost level and, thus, raise expected participation levels. That would imply $\max c^{*} \leq c^{\text {opt }}$. The ex ante unconditional utility of any agent in a mechanism $c(\cdot)$ with interior participation probability $\tau=F(c)$ is

$$
\begin{aligned}
U_{i}(c) & =\int_{\underline{c}}^{\bar{c}} U_{i}\left(c(\cdot) \mid c_{i}\right) d F\left(c_{i}\right) \\
& =\int_{\underline{c}}^{c} U_{i}\left(c(\cdot) \mid c_{i}\right) d F\left(c_{i}\right)+\int_{c^{*}}^{\bar{c}} U_{i}\left(c(\cdot) \mid c_{i}\right) d F\left(c_{i}\right) \\
& =F(c) u_{p}(c)-\int_{\underline{c}}^{c} c_{i} d F\left(c_{i}\right)+[1-F(c)] u_{a}(c),
\end{aligned}
$$

where $u_{a}(c)$ is the ex ante unconditional utility of abstention as in equation 13 and $u_{p}(c)$ is the ex ante unconditional utility of participation in such a mechanism as in equation 14 . Note that as before both these unconditional utilities are positively increasing in $\tau$, i.e., $u_{a}^{\prime}(\tau)>0$ and $u_{p}^{\prime}(\tau)>0$.

Now let us take the first derivative of ex ante utility $U_{i}\left(c^{*}\right)$ (first-order condition) and compute its value for any Bayesian Nash equilibrium cutoff $c^{*}$ :

$$
\begin{aligned}
U_{i}^{\prime}\left(c^{*}\right) & =F\left(c^{*}\right) u_{p}^{\prime}\left(c^{*}\right)+f\left(c^{*}\right) u_{p}\left(c^{*}\right)-c^{*} f\left(c^{*}\right)+\left[1-F\left(c^{*}\right)\right] u_{a}^{\prime}\left(c^{*}\right)-f\left(c^{*}\right) u_{a}\left(c^{*}\right) \\
& =F\left(c^{*}\right) u_{p}^{\prime}\left(c^{*}\right)+\left[1-F\left(c^{*}\right)\right] u_{a}^{\prime}\left(c^{*}\right)+f\left(c^{*}\right)\left(u_{p}\left(c^{*}\right)-u_{a}\left(c^{*}\right)-c^{*}\right) \\
& =F\left(c^{*}\right) u_{p}^{\prime}\left(c^{*}\right)+\left[1-F\left(c^{*}\right)\right] u_{a}^{\prime}\left(c^{*}\right)>0 .
\end{aligned}
$$

The last step follows from the fact that in an interior Bayesian equilibrium $B\left(F\left(c^{*}\right)\right)=c^{*}$. Since this will hold for all $i \in N$ in a symmetric equilibrium, then it follows that any interior equilibrium cutoff $c^{*} \in(\underline{c}, \bar{c})$ will be ex ante Pareto inefficient and an increase in the cutoff 
point is locally Pareto-improving in all such equilibria.

To complete the proof, we need to examine boundary cutoff equilibria $\underline{c}$ and $\bar{c}$ and show that if $c^{*}=\bar{c}$ then $c^{o p t}=\bar{c}$ and if $c^{*}=\{\underline{c}\}$ then $c^{o p t} \geq \underline{c}$. In the first case, the derivative function from equation 17 becomes $U_{i}^{\prime}(\bar{c})=u_{p}^{\prime}(\bar{c})+f\left(c^{*}\right)\left(u_{p}\left(c^{*}\right)-u_{a}\left(c^{*}\right)-c^{*}\right)>0$, since $B(1) \geq \bar{c}$; hence, any strategically equivalent $c^{o p t} \geq \bar{c}$ will constitute an ex ante Pareto efficient cutoff mechanism. In the second case where $\underline{c}$ is the unique equilibrium cutoff, depending on the magnitude of $B(0)-\underline{c}$ it may be that $c^{\text {opt }}>\underline{c}$, otherwise it is trivially assumed that $c^{o p t}=\underline{c}$ (since all monotonic cutoff mechanisms $c \leq \underline{c}$ are strategically equivalent for a given support of cost types $[\underline{c}, \bar{c}])$. Q.E.D.

In order to induce optimally higher participation, the mechanism designer (or external benefactor) needs to subsidize participation costs within the community. ${ }^{20}$ Given that $\max c^{*} \leq c^{\text {opt }}$, he/she will therefore set the ex ante level of the optimal unit subsidy $s^{*}$ such that an agent with individual cost $c^{\text {opt }}$ becomes just indifferent between participating or not, that is the incentive compatibility constraint becomes just binding. ${ }^{21}$ Hence, $s^{*}=c^{o p t}-B\left(F\left(c^{o p t}\right)\right) \cdot{ }^{22}$ On a related point, the existence of multiple equilibria also implies a task of equilibrium selection for the mechanism designer. One can think of a case where $c^{\text {opt }}$ is indeed a Nash equilibrium, but the community has been trapped in a suboptimally low participation equilibrium of $c^{* s u b}$. Under this scenario it would be advisable for the benevolent dictator to forcefully coerce the community into the better equilibrium, or otherwise to create strong focal points that will attract attention to optimal levels of participation.

\footnotetext{
${ }^{20}$ In other words, he/ she needs to induce agents to internalize the social benefits of their participation, which are $N$ times their private benefits. It is in this sense that the quality of the decision-making process is like a public good.

${ }^{21} \mathrm{~A}$ monotonic cutoff mechanism $\widetilde{c}(\cdot)$ in this Bayesian collective-choice problem is interim incentive compatible if and only if $U_{i}\left(\widetilde{c}(\cdot) \mid c_{i}\right) \geq U_{i}^{*}\left(\widetilde{c}(\cdot), c_{i}^{\prime} \mid c_{i}\right)$ for all $i \in N, c_{i} \in[\underline{c}, \bar{c}]$, and $c_{i}^{\prime} \in[\underline{c}, \bar{c}]$. This holds for Bayesian Nash equilibrium cutoff points $\widetilde{c}=c^{*}$, since $B\left(F\left(c^{*}\right)\right)=c^{*}$. As a result, $c^{*}(\cdot)$ 's are interim and ex post Pareto efficient mechanisms, but not ex ante efficient.

${ }^{22}$ Note that the assumption of an external social planner obviates the social need to balance the budget. Hence, concerns of an implementable mechanism that balances the budget do not arise here.
} 
In this context, mechanism design amounts to creating incentives for an optimal increase in participation through a uniform transfer scheme of subsidies, which explains why it appears more conducive to take a public goods approach to mechanism design. Regardless of the formulation of the problem, whether stochastic, heterogeneous or with complete information, the quality of the decision (i.e., the probability of errors) is a public good in the sense that it affects everyone's utility equally, while its cost is borne differentially by jury members. Freeriding arises in the Bayesian equilibrium because agents do not internalize the marginal social benefits of participation in the decision-making process. There is a positive informational externality that agents do not take into account once left to their own means to interact with each other. This is why voluntary symmetric Nash equilibria are socially inefficient.

\section{Discussion}

I have presented a simple model of optimal committee design within a small community of finite size. The model can be generalized into a whole class of common value problems. Building on the theoretical framework of the seminal papers on information aggregation, I have derived the optimal size of a jury based on the assumption of informative voting (that helps surmount the dual optimization difficulty) and, subsequently, compared that to various symmetric equilibria that may arise in a complete information setting or a Bayesian environment with heterogeneous private costs of participation. I also sketch out an optimal transfer scheme that may implement the ex ante socially efficient political participation mechanism.

One possible extension would be to assume a governing body within the community that administers the transfer scheme, but is also expected to balance the budget. Upon introducing heterogeneity in preference intensities (types $q_{i}$ ) one may arguably use the results of the mechanism design literature on the optimal provision of public goods (see for example Bergstrom, Blume and Varian, 1986; Bernheim, 1986; Bliss and Nalebuff, 1984; Groves and 
Ledyard, 1977; Ledyard and Palfrey, 2002), in order to conduct an implementation analysis of optimal committee design under a balanced-budget transfer scheme.

One other interesting extension to the model would amount to incorporating a private values component in the utility functions and finding the relation between the utility weight on that component and optimal committee size. Alternatively, one could postulate the existence of partisans or biased jurors, who derive private utility gains from either solution to the binary problem regardless of their information about the state of the world, thus unveiling a larger set of political participation mechanisms. ${ }^{23}$ This extended version of the model would certainly have something of import to say on the comparison between the selection mechanisms of juries and legislatures. It could provide a rationale for the use of different selection mechanisms in these two branches of state authority, which basically differ in the relative weights of the common value and private value components of their respective collective choice domains (the former predominates in the judiciary and the latter in the legislature). What if juries were elected and legislators randomly drawn from a pool of citizens (see for example Bergstrom and Varian, 1984)?

Last but not least, the above framework may be enriched in order to study notions of jury bias or representativeness by allowing for different priors or a correlation between individual costs and signal informativeness. What is essentially a 'jury of one's peers'? What kinds of mechanisms minimize the bias against the defendant and maximize social welfare at the same time? These are questions whose study falls within the scope of an extended theoretical framework.

\footnotetext{
${ }^{23}$ See ? for a jury model with preference diversity among jurors and ? for a model of committee size with preference heterogeneity and costly participation.
} 


\section{Appendix}

Proof of Proposition 2. We need to show that $\lim _{N \rightarrow \infty} n^{*}(N)=\bar{n}^{*}<\infty$, or else that the monotonically increasing sequence $\left\{n^{*}(N)\right\}, N=0,1,2, \ldots$ has a finite upper bound. One of the properties of this sequence is that the absolute difference between any two of its elements is an even number. Let us look at a related sequence $\left\{\underline{N}_{n_{0}+2 l}\right\}$, where $l=0,1,2, \ldots$, $n_{0}=\min \left\{n^{*}(N): n^{*}(N)>0\right\}$, and $\underline{N}_{n_{0}+2 l}=\min \left\{N: n^{*}(N)=n_{0}+2 l\right\}$. This sequence lists the consecutive minimum population sizes required to raise the optimal jury size by 2 .

The proof proceeds by showing that the limit of this sequence is infinite. Fix some infinitesimal $\varepsilon>0$ and some finite positive integer $\bar{l}_{\varepsilon}$ such that $n_{\bar{l}_{\varepsilon}}=n_{0}+2 \bar{l}_{\varepsilon}>n_{\varepsilon}$, where $n_{\varepsilon}$ is such that for all $n>n_{\varepsilon},\left|A M B\left(n, k^{*}(n)\right)\right|<\varepsilon(\dagger)$ (making use of the fact that the $A M B(\cdot, \cdot)$ function converges to zero). Now pick some $l^{\prime}=\bar{l}_{\varepsilon}+j$ for any $j=1,2, \ldots$ Then $\underline{N}_{n_{0}+2 l^{\prime}}=\min \left\{N^{\prime}: n^{*}\left(N^{\prime}\right)=n_{0}+2 l^{\prime}\right\}$ and it necessarily has to be the case that $E W\left(n_{l^{\prime}}, k^{*}\left(n_{l^{\prime}}\right) ; N^{\prime}\right)>E W\left(n_{\bar{l}_{\varepsilon}}, k^{*}\left(n_{\bar{l}_{\varepsilon}}\right) ; N^{\prime}\right) \Leftrightarrow N^{\prime} \sum_{n=n_{\bar{l}_{\varepsilon}}}^{n_{\bar{l}_{\varepsilon}}+2 j} A M B\left(n, k^{*}(n)\right)>2 j c$. From $(\dagger)$ above it must also be that $2 j \varepsilon N^{\prime}>N^{\prime} \sum_{n=n_{\bar{l}_{\varepsilon}}}^{n_{\bar{l}_{\varepsilon}}+2 j} A M B\left(n, k^{*}(n)\right)>2 j c$. But for this inequality $\varepsilon N^{\prime}>c>0$ to hold for $\varepsilon \rightarrow 0$, it must be that $N^{\prime} \rightarrow \infty$, and, therefore, $\underline{N}_{n_{0}+2 \bar{l}_{\varepsilon}} \underset{\varepsilon \rightarrow 0}{\longrightarrow} \infty$. This in turn implies that the original sequence $\left\{n^{*}(N)\right\}$ converges to some limit point $n_{\bar{l}}$. By theorem 3.13 in Rudin (1976, p. 55), a monotonically increasing sequence converges if and only if it is bounded by above, hence $\left\{n^{*}(N)\right\}$ has a finite upper bound. Q.E.D.

\section{References}

Austen-Smith, David and Jeffrey S. Banks. 1996. "Information Aggregation, Rationality, and the Condorcet Jury Theorem." American Political Science Review 90(1):34-45.

Austen-Smith, David and Timothy J. Feddersen. 2005. Social Choice and Strategic Decisions: 
Essays in Honor of Jeffrey S. Banks. In Social Choice and Strategic Decisions: Essays in Honor of Jeffrey S. Banks, ed. David Austen-Smith and John Duggan. Berlin; Heidelberg: Springer pp. 269-316.

Bergstrom, Theodore C. and Hal R. Varian. 1984. "Government by Jury." Mimeo, Department of Economics, University of Michigan.

Bergstrom, Theodore C., Lawrence Blume and Hal R. Varian. 1986. "On the Private Provision of Public Goods." Journal of Public Economics 29:25-49.

Bernheim, Douglas B. 1986. "On the Voluntary and Involuntary Provision of Public Goods." American Economic Review 76(4):789-793.

Bliss, Christopher and Barry Nalebuff. 1984. "Dragon Slaying and Ballroom Dancing: The Private Supply of a Public Good.” Journal of Public Economics 25(1-2):1-12.

Bulkley, George, Gareth D. Myles and Bernhard R. Pearson. 2001. "On the Membership of Decision-Making Committees." Public Choice 106(1-2):1-22.

Börgers, Tilman. 2004. "Costly Voting." American Economic Review 94(1):57-66.

Caillaud, Bernard and Jean Tirole. 1999. "Party Governance and Ideological Bias." European Economic Review 43(4-6):779-789.

Caillaud, Bernard and Jean Tirole. 2002. "Parties as Political Intermediaries." Quarterly Journal of Economics 117(4):1453-1489.

Coughlan, Peter J. 2000. "In Defense of Unanimous Jury Verdicts: Mistrials, Communication, and Strategic Voting." American Political Science Review 94(2):375-393.

Duggan, John and Cesar Martinelli. 2001. "A Bayesian Model of Voting in Juries." Games and Economic Behavior 37(2):259-294. 
Feddersen, Timothy J. and Wolfgang Pesendorfer. 1996. "The Swing Voter's Curse." American Economic Review 86(3):408-424.

Feddersen, Timothy J. and Wolfgang Pesendorfer. 1997. "Voting Behavior and Information Aggregation in Elections with Private Information.” Econometrica 65:1029-1058.

Feddersen, Timothy J. and Wolfgang Pesendorfer. 1998. "Convicting the Innocent: The Inferiority of Unanimous Jury Verdicts under Strategic Voting.” American Political Science Review 92(1):23-35.

Ghosal, Sayantan and Ben Lockwood. 2003. Information Aggregation, Costly Voting and Common Values. The Warwick Economics Research Paper Series (TWERPS) 670 University of Warwick, Department of Economics.

Groves, Theodore and John O. Ledyard. 1977. "Optimal Allocation of Public Goods: A Solution to the Free-Rider Problem." Econometrica 45(4):783-809.

Hafer, Catherine and Dimitri Landa. 2007. "Deliberation as Self-discovery and Institutions for Political Speech." Journal of Theoretical Politics 19(3):329-360.

Ladha, Krishna K. 1992. "The Condorcet Jury Theorem, Free Speech, and Correlated Votes." American Journal of Political Science 36(3):617-634.

Ledyard, John O. and Thomas R. Palfrey. 2002. "The Approximation of Efficient Public Good Mechanisms by Simple Voting Schemes." Journal of Public Economics 83(2):153-171.

McLennan, Andrew. 1998. "Consequences of the Condorcet Jury Theorem for Beneficial Information Aggregation by Rational Agents.” American Political Science Review 92(2):413418. 
Meirowitz, Adam. 2006. "Designing Institutions to Aggregate Preferences and Information." Quarterly Journal of Political Science 1(4):373-392.

Meirowitz, Adam. 2007. "In Defense of Exclusionary Deliberation: Communication and Voting with Private Beliefs and Values." Journal of Theoretical Politics 19(3):301-327.

Myerson, Roger B. 1997. Game Theory: Analysis of Conflict. Cambridge, MA: Harvard University Press.

Myerson, Roger B. 1998a. "Extended Poisson Games and the Condorcet Jury Theorem." Games and Economic Behavior 25(1):111-131.

Myerson, Roger B. 1998b. "Population Uncertainty and Poisson Games." International Journal of Game Theory 27(3):375-392.

Palfrey, Thomas R. and Howard Rosenthal. 1983. "A Strategic Calculus of Voting." Public Choice 41(1):7-53.

Palfrey, Thomas R. and Howard Rosenthal. 1985. "Voter Participation and Strategic Uncertainty." American Political Science Review 79(1):62-78.

Persico, Nicola. 2004. "Committee Design with Endogenous Information." Review of Economic Studies 71(1):165-191.

Rudin, Walter. 1976. Principles of Mathematical Analysis. 3rd ed. Singapore: McGraw-Hill. 